\title{
Outcome and rehabilitation of patients following aneurysmal subarachnoid haemorrhage
}

\author{
Dhaval P. Shukla
}

\begin{abstract}
Aneurysmal subarachnoid haemorrhage (SAH) is a dreaded neurosurgical disorder. Although the mortality has been declining in the past three decades, the disability after SAH is still significant. The main determinants of outcome are age, clinical grade, amount of SAH and size of aneurysm. Both neurophysical deficits and neuropsychological impairments are determinants of functional outcome after SAH. Patients should be assessed using outcome measures for both traumatic brain injury and stroke. Early rehabilitation after SAH improves not only physical outcome but also cognitive and functional outcome.
\end{abstract}

Key words: Cognitive, neuropsychological, outcome, rehabilitation, subarachnoid haemorrhage

\section{INTRODUCTION}

Aneurysmal subarachnoid haemorrhage (SAH) is a lethal disease. ${ }^{[1]}$ In recent years, the case-fatality rate after SAH has decreased. After adjustment for age and sex the decrease in case fatality is $0.9 \%$ / year during the past three decades. ${ }^{[2,3]}$ However, SAH is still one of the most feared neurologic disorders. Among the survivors, up to two-third have a significantly reduced quality of life (QoL) with 30\% of these patients remaining dependent. ${ }^{[4]}$ An estimated lifetime cost is more than double that of an ischaemic stroke. In SAH International Trialists (SAHIT) repository comprising nearly 10,000 patients, the outcomes of treated SAH at 3 months as assessed using Glasgow Outcome Scale (GOS) were as following: Good recovery $50 \%$, moderate disability $21 \%$, severe disability $14 \%$, vegetative state $3 \%$ and death $12 \% .{ }^{[5]}$

Department of Neurosurgery, National Institute of Mental Health and Neurosciences, Bengaluru, Karnataka, India

Address for correspondence:

Dr. Dhaval P. Shukla, Department of Neurosurgery, National Institute of Mental Health and Neurosciences, Bengaluru - 560 029, Karnataka, India. E-mail: neurodhaval@rediffmail.com

\begin{tabular}{|l|l|}
\hline \multicolumn{2}{|c|}{ Access this article online } \\
\hline Quick Response Code: & Website: \\
\hline & www.jnaccjournal.org \\
\hline & \\
\hline
\end{tabular}

\section{DETERMINANTS OF OUTCOME}

Several factors influence outcome after SAH. These are patient factors such as age, medical comorbidities; aneurysm factors such as severity of SAH and posterior circulation aneurysms; and treatment factors like time to treatment, Neurointensive Care Unit (NICU) facilities, endovascular services and volume of SAH treated. Where the patient is treated has influence on the outcome. Neurointensivist care in a NICU improves patient survival and outcomes. ${ }^{[6,7]}$ In a large randomised study (the International Subarachnoid Aneurysm Trial) in which a modified Rankin Scale (MRS) was used, the mortality rate and dependency were comparable between surgical and endovascular therapy for aneurysms associated with SAH. ${ }^{[8]} \mathrm{A}$ centre that offers both treatments, that is, microsurgical and endovascular is likely to have better outcome. ${ }^{[8]}$

A number of parameters have been used to develop prediction models of outcome after SAH. Among them the most consistent predictors of outcome are age, clinical grade, SAH grade on computed tomography (CT) scan and aneurysm size. ${ }^{[9]}$

This is an open access article distributed under the terms of the Creative Commons Attribution-NonCommercial-ShareAlike 3.0 License, which allows others to remix, tweak, and build upon the work non-commercially, as long as the author is credited and the new creations are licensed under the identical terms.

For reprints contact: reprints@medknow.com

How to cite this article: Shukla DP. Outcome and rehabilitation of patients following aneurysmal subarachnoid haemorrhage. J Neuroanaesthesiol Crit Care 2017;4:S65-75. 


\section{Age}

There is a linear association between advancing age and worse outcome. The odds ratio of 60 days mortality is 1.32 for each decade increase in age. ${ }^{[10]}$ The risk of poor outcome is significantly increased after the age of 60 years. ${ }^{[11]}$ Aging patients have a higher probability of dying or sustaining permanent neurologic damage. In the study by Lanzino et al. the complication rate increased from $28 \%$ in the sub- 40 -year group to $46 \%$ in the patients older than 70 years. Mortality rates are also worse for older patients (35\% in the $>70$-year group) as compared with younger patients $(12 \%$ in the $<40$-year group) with the same clinical presentation. Patients aged 40 years or younger made a good recovery $73 \%$ of the time, with the same degree of recovery found in only $25 \%$ of patients aged 70 years or older. Older patients were more likely to have hydrocephalus and showed an increased rehaemorrhage rate $(4.5 \%$ in the sub-40-year group to $16.4 \%$ in the above 70 -year group). ${ }^{[2]}$ Older patients are more likely to present with a lower level of consciousness, have a poorer World Federation of Neurosurgical Societies (WFNS) score, have a thicker subarachnoid blood load, and are more likely to have associated intraventricular blood. In older patients, increased systolic blood pressure is more common and is associated with a poorer WFNS grade. Advancing age is also associated with increased comorbidities, including hypertension, diabetes, pulmonary dysfunction, cardiac disease and cerebrovascular disease. The aging brain seems to be less able to cope with the secondary effects of a SAH. The reasons are a combination of factors, ranging from structural changes, biochemical and reduced plasticity. ${ }^{[11]}$

\section{Clinical grade}

In SAHIT repository favourable outcome was seen in $82 \%$ of patients in good grade (WFNS I and II) as compared to only $39 \%$ of patients in poor grade (WFNS IV and V). The mortality was $5.9 \%$ in patients with good grade and $35.3 \%$ in patients with poor grade. ${ }^{[13]}$ Patients in WFNS Grade 2SAH are likely to have 1.87 times risk of mortality, and patients in WFNS Grade 5 are likely to have 7 times risk of mortality as compared to patients in WFNS Grade 1. ${ }^{[10]}$

\section{Computed tomography scan grade}

In SAHIT repository the mortality was $5 \%$ in patients with Fisher Grade $1 \mathrm{SAH}$, and $12.7 \%$ in patients with Fisher Grade 4 SAH. The outcome was favourable in $89.8 \%$ patients with Fisher Grade $1 \mathrm{SAH}$, whereas the outcome was favourable in only $62.6 \%$ of patients with Fisher Grade 4 SAH. ${ }^{[5]}$ Patients with Fisher Grade $2 \mathrm{SAH}$ on CT scan have 1.43 times more risk, and with Fisher Grade 4, 2.76 more risk of dying at 2 months after SAH as compared to patients with Fisher Grade 1. ${ }^{[10]}$ Higher Fisher grades are associated with worse neurological status at presentation and increased risk of cerebral vasospasm. Hence, the mortality is more and favourable outcome is less in patients with higher Fisher grade.

\section{Size of aneurysm lumen}

The aneurysm diameter has a U-shaped relationship with outcome, a finding which suggests worse outcomes at extremes of size, that is, $<4$ or $>9 \mathrm{~mm}$. Very small aneurysms are associated with more extensive haemorrhage, are more difficult to treat, are associated with a relatively higher risk of periprocedural complications, and have relatively higher morbidity and mortality. Giant aneurysms may present similar challenges.${ }^{[5]}$ For each $\mathrm{mm}$ increase in size of aneurysm the odds ratio of 60 day mortality is $1.08 .^{[10]}$

\section{AREAS OF DYSFUNCTION AFTER SUBARACHNOID HAEMORRHAGE}

The pathophysiology of brain injury after SAH is quite similar to traumatic brain injury (TBI) and stroke. The end result of pathophysiological mechanism after SAH is focal and scattered brain injury due to ischaemia occurring either during the initial haemorrhage or as a consequence of macro- and micro-vascular dysfunction and delayed cerebral ischaemia (DCI). [4] The combination of focal and scattered brain injury results in cognitive impairment and/or focal neurological deficits. The common dysfunction seen in survivors of SAH involve domain of neurophysical functions like motor and sensory deficits; and cognitive functions such as memory, executive function, language and visuospatial.

\section{NEUROPHYSICAL IMPAIRMENT}

Neurological deficits are not uncommon after SAH. Neurological deficits can occur due to haemorrhage itself, vasospasm, or the result of treatment. ${ }^{[14]}$ Earlier studies have found that the neurological impairment after SAH improves over time and is not important in determining the outcome. ${ }^{[15,16]}$ However, neurological deficits are determinant of functional outcome. In the Intraoperative Hypothermia for Aneurysm Surgery Trial, post-operative neurological deficits were observed in $42.6 \%$ of the patients. ${ }^{[17]} \mathrm{New}$ focal motor deficit accounted for $65 \%$ of post-operative neurological deterioration, whereas $60 \%$ was accounted for using the National Institute of Health and Stroke Scale (NIHSS) total score change (>4-point) and 51\% by Glasgow Coma Scale score change. Following factors were significantly associated with occurrence of post-operative neurological deterioration: age, Fisher grade on admission, occurrence of a procedure before aneurysm surgery (ventriculostomy), timing of surgery, systolic blood pressure during surgery, ST segment 
depression during surgery, history of abnormality in cardiac valve function, use of intentional hypotension during surgery, duration of anterior cerebral artery occlusion, intraoperative blood loss and difficulty of aneurysm exposure. Although the NIHSS scores improved over 3 months, still presence of neurological deficits was the major determinant of outcome. In that study, only $46.2 \%$ of patients with neurological deficits had a good recovery as determined by GOS, whereas $77.7 \%$ of patients without post-operative neurological deterioration had a good recovery $(P<0.05) \cdot{ }^{[17]}$ In another study, abbreviated NIHSS scores at follow-up was significantly found to affect the outcome. ${ }^{[18]}$ Neurological injury incurred perioperatively or in the acute post-operative period accounts for a large percentage of poor outcomes in patients with good admission WFNS grades undergoing surgery for SAH.

\section{NEUROPSYCHOLOGICAL (COGNITIVE) IMPAIRMENT}

Although cognitive deficit after SAH is well known, the neuropsychological outcome is underreported. In a review of studies on outcome after $\mathrm{SAH}$, only $6.5 \%$ of studies reported the neuropsychological outcome. ${ }^{[19]}$ Frequently impaired cognitive domains in patients with SAH are the memory, executive function, and language. Verbal memory is one of the most frequently impaired cognitive domains, with the prevalence of impairment ranging from $14 \%$ to $61 \%$ in patients with SAH. ${ }^{[20]}$ Executive functions like planning, inhibition, strategizing, problem solving, attention, and decision making are impaired in 3\%-76\%. The prevalence of language impairment in $\mathrm{SAH}$ is $0 \%-76 \%{ }^{[21]}$ The variable results across studies are attributed to the timing of assessment, the definition of impairment, and the tests used. The time course of cognitive recovery after SAH is heterogeneous. ${ }^{[2]}$ The neuropsychological evaluation has to be performed beyond 3 months after $\mathrm{SAH}$ as patients are likely to improve with time. ${ }^{[22]}$ Another problem with studies on neuropsychological assessment is the definition of impairment. Most cognitive tests do not have a validated threshold for impairment, the majority of studies define impaired performance using arbitrary cut-offs. Usually, impaired performance is defined as performance $<2$ standard deviations (SDs) relative to population norms. However, not all studies adopt this cut-off. ${ }^{[20]}$ A more generous cut-off coincides with a greater prevalence of cognitive impairment. Variable cut-offs of neuropsychological scores may partially account for the wide range of dysfunction in different cognitive domains. To obviate this confounding factor of cut-off, one can use absolute neuropsychological scores to correlate with outcome. ${ }^{[23]}$ Besides the cut-off, age-associated cognitive decline can be responsible for observed worse neuropsychological scores in patients with SAH. ${ }^{[4]}$ The two decades between 50 and 70 years of age are associated with noticeable effects on memory and learning. This period also happens to be when SAH occurs. If patients with $\mathrm{SAH}$ are tested 10 years after haemorrhage, the patients will most likely exhibit cognitive decline simply because they have aged 10 years. To dampen this confounding factor a control group that is age, gender and level of education matched should be used to assess cognitive impairment in patients with SAH. The third problem with studies on neuropsychological assessment is a lack of agreement on neuropsychological tests used for patients with brain injury. ${ }^{[25]}$ There is no consensus for the type of neuropsychological test battery required for assessment of cognitive impairment in patients with brain injury. ${ }^{[25]}$ Comprehensive neuropsychological assessment, although providing valuable information about the cognitive function, may not be suitable for all patients after SAH. Over $28 \%$ of survivors are incapable of completing neuropsychological tests because of test difficulty, poor clinical condition, or severe headache from mental exercise. ${ }^{[2]}$ We have used a tailored test battery from our institute's comprehensive neuropsychological battery. ${ }^{[23,26]}$ The tests used by us covered major domains of cognitive impairments seen in patients with SAH [Table 1]. This customised battery can be completed within $60 \mathrm{~min}$, and can be administered by non-neuropsychologists with some training. Cognitive performance can be affected by post-SAH symptoms, headache, depression, fatigue, sleep disorders, etc. Studies on cognitive outcome may overlook neurological deficits such as motor weakness, vision impairment, etc., as confounding factor. Wong et al. took into consideration confounding factors, neurological impairment and depression, to assess cognitive functions. ${ }^{[18]}$ The authors found that unfavourable outcome was significantly

\section{Table 1: Neuropsychological tests used for assessment in patients with subarachnoid haemorrhage}

Tests of attention and working memory (frontoparietal networks)

$$
\begin{aligned}
& \text { Digit span (Wechsler's Memory Scale 3) } \\
& \text { Spatial span (Wechsler's Memory Scale 3) }
\end{aligned}
$$

Test for orbitofrontal and anterior cingulate cortex

Response inhibition (Stroop test)

Test for prefrontal cortex

Trail making test

Controlled Oral Word Association test

Test for verbal and visual learning and memory (left and right temporal lobe)

Rey's auditory verbal learning test

Rey complex figure test 
associated with the presence of cognitive deficits, abbreviated NIHSS score, and Geriatric Depression Scale (GDS) scores. The unfavourable outcome was also significantly associated with cognitive deficits after adjustments of abbreviated NIHSS score and GDS score. This indicates that functional outcome after SAH is affected by both neurological and cognitive deficits, and cognitive deficits irrespective of neurological deficits also affect the outcome. Among the cognitive functions attention and working memory are main determinants of functional outcome after SAH. ${ }^{[23]}$

Aneurysm of the anterior communicating artery (ACoA) has been extensively reviewed and specific neurobehavioural and neuroanatomic sequelae described. ${ }^{[27]}$ ACoA syndrome has been defined as amnesia, confabulation and personality change, although this exact pattern is not required. The frontal structures are implicated in producing this syndrome. However, it is controversial whether this syndrome is specific to $\mathrm{ACoA}$ or it is due to SAH in general. ${ }^{[27]}$

\section{NEUROPSYCHIATRIC SYMPTOMS AFTER SUBARACHNOID HAEMORRHAGE}

The frequent neuropsychiatric diagnosis made in SAH patients are depression (45\%), apathy (42\%), denial (21\%) and catastrophic reaction (17\%). ${ }^{[28]}$ Over $31 \%$ of patients report feeling tired on a daily basis. Sleep is often impaired in $37 \%-45 \%$ of patients. Sleep efficiency and daytime function are significantly below normal levels. Anxiety affects up to $54 \%$ of patients. At 3 months, $60 \%$ of patients report intrusive thoughts or avoidance of reminders, consistent with post-traumatic stress disorder.

Depression is often over looked psychiatric sequelae after any brain injury. The symptoms of depression are identical to frontal lobe syndrome (impaired concentration, reduced fluency of speech, apathy, inattentiveness, delayed responses to questions; and striking lack of inhibition, including socially inappropriate behaviour [anger, agitation, aggression]). Frontal lobe syndrome and depression can overlap in patients with SAH, whereby depression is seen as part of the frontal lobe syndrome. Unlike cognitive domains that improve over time depression remains stable over the 18-month period after SAH.

\section{FUNCTIONAL OUTCOME AFTER SUBARACHNOID HAEMORRHAGE}

Activities of daily living (ADL) of self-care such as feeding, grooming, dressing, bathing, and toileting are deficient in long-term in up to $12 \%$ of patients. Instrumental ADL of more complex tasks like managing finances, shopping, transport, medication management, housekeeping, etc., are deficient in upto $93 \%$ patients. About $40 \%-80 \%$ of patients never return to previous occupation.

\section{OUTCOME ASSESSMENT TOOLS FOR SUBARACHNOID HAEMORRHAGE}

As sequelae after SAH has a pattern similar to that of TBI and ischaemic stroke, a few outcome assessment tools used for these two diseases are suitable for use in survivors of SAH. ${ }^{[25,29]}$ The outcome measures that can be used for SAH can be classified according to the World Health Organization (WHO) International Classification of Functioning domains. These domains are following.

\section{Imapairment}

Imapairment, defined as any loss or abnormality of psychological, physiological, or anatomical structure or function. Quantification of neurological deficits (impairment) can be done using NIHSS score. The NIHSS tests following 11 neurological functions:

1. Level of consciousness

2. Gaze

3. Visual field

4. Facial palsy

5. Motor function arms

6. Motor function legs

7. Limb ataxia

8. Sensory

9. Language

10. Dysarthria

11. Extinction and inattention.

The NIHSS has total scores ranging from 0 to 42 with higher values representing more severe deficits. ${ }^{[30]}$ A 4-point worsening in NIHSS score is considered statistically significant. The impairment of neurological function can be classified as following based on NIHSS scores:

- $\quad>25$ : Very severe neurological impairment

- 15-24: Severe impairment

- 5-14: Moderately severe impairment

- $\quad$ 5: Mild impairment.

\section{Activity limitation}

Activity limitation, defined as any restriction or lack (resulting from an impairment) of ability to perform an activity in the manner or within the range considered normal for a human being. MRS, Barthel index (BI), and functional independence measure (FIM), are the most common scales used to assess activity limitation due to stroke. ${ }^{[31]}$

The MRS measures independence rather than performance of specific tasks. In this way, mental as well as physical adaptations to the neurological deficits 
are incorporated. The scale consists of six grades, from 0 to 6 , with 0 corresponding to no symptoms and 5 corresponding to severe disability, and 6 to death. The cut-off for defining poor outcome is $>3$ (moderate disability; requiring some help, but able to walk without assistance) ${ }^{[32]}$ This simple scale is widely used but is very crude, with low sensitivity for detecting between-group differences. The MRS has been criticised for mixing impairment, activity, and participation aspects of recovery, but is most often categorised as a global measure of activity. ${ }^{[29]}$

The BI is crude but simple instrument. The BI measures the patient's performance in 10 activities of daily life. The BI is considered a reliable disability scale for stroke patients. ${ }^{[32]}$ The items can be divided into a group that is related to self-care (feeding, grooming, bathing, dressing, bowel and bladder care, and toilet use) and a group related to mobility (ambulation, transfers, and stair climbing). The maximal score is 100 indicating that the patient is fully independent in physical functioning. The lowest score is 0 , representing a totally dependent bedridden state. The cut-off for defining poor outcome is $\mathrm{BI}<60 .{ }^{[32]}$ The BI was the major outcome assessment instrument in use in rehabilitation. It was improved on and supplanted by the FIM. It has been found inferior to FIM.

The FIM is the most widely accepted functional assessment measure in use in the rehabilitation community. It is viewed as most useful for assessment of progress during inpatient rehabilitation. The FIM is an 18-item ordinal scale, used with all diagnoses within a rehabilitation population. Two separate domains of items are: The motor domain consisting of 13 items and the cognitive domain consisting of 5 item. The FIM measures independent performance in self-care, sphincter control, transfers, locomotion, communication, and social cognition. FIM scores range from 1 to 7: A FIM item score of 8 is categorized as 'complete independence', while a score of one is 'total assistance' (performs $<25 \%$ of task). Scores falling below six require another person for supervision or assistance. By adding the points for each item, the possible total score ranges from 18 (lowest) to 126 (highest) level of independence. ${ }^{[33]}$ It has been used to assess progress of rehabilitation in patients with $\mathrm{SAH} .{ }^{[34]}$

\section{Participation restriction}

Participation restriction, defined as a disadvantage for a given individual, resulting from an impairment or a disability, that limits or prevents the fulfilment of a role that is normal (depending on age, sex, and social and cultural factors) for that individual. In 1988, WFNS proposed use of GOS for assessment of functional outcomes after SAH. ${ }^{[35]}$ Although the GOS has been utilized extensively, it has been criticized as suffering from ceiling effects and being insufficiently sensitive to subtle but functionally limiting deficits in cognition, mood and behaviuor. Disproportionate weight of the physical (in comparison to the cognitive and emotional) deficits has been measured in many studies. These limitations in the GOS create ambiguity in the assessment of patients who have achieved a good recovery but have not returned to preinjury status. To address these shortcomings, the GOS has been modified and a structured interview has been proposed to accurately categorise patient's disability. This version is called the GOS-extended (GOSE). ${ }^{[36]}$ The GOSE has total eighth categories (1-8), lowest value 1 indicates death and highest value 8 indicates upper level of good recovery. GOSE has been dichotomised for ease of statistical analysis. The favourable outcome includes good recovery (upper and lower) and moderate disability (upper and lower). The unfavourable outcome includes severe disability (upper and lower), vegetative state, and death.

QoL is a broad and ill-defined concept. QoL has been taken to be synonymous with health status, physical functioning, perceived health status, subjective health, health perception, symptoms, need satisfaction, individual cognition, functional disability, psychiatric disturbance, well-being, and often, several of these at the same time. ${ }^{[37]}$ Short form-36 and sickness impact profile have been used to assess QoL in survivors of SAH. ${ }^{[2]}$

\section{REHABILITATION AFTER SUBARACHNOID HAEMORRHAGE}

Rehabilitation is the combined and co-ordinated efforts of a physician supervised multi-disciplinary team in helping a diseased person to reach maximum physical, psychological, social, vocational and educational potential, consistent with his or her physiological or anatomical impairment, environmental limitations, desires and life plans. Although the guidelines for management of SAH have been published, it is interesting to note that there is no mention about rehabilitation. The principles of rehabilitation are similar to that for TBI and stroke. The practical tips given below are taken from guidelines for rehabilitation in TBI. ${ }^{[38]}$

The old paradigm was that rehabilitation is the third phase of medicine. The new paradigm is that rehabilitation should be initiated as early as possible. The basis of this paradigm shift is better understanding of brain plasticity. There are practice related changes in functional activations, redistribution and reorganisation of areas of brain. ${ }^{[39]}$ Overtime there is recruitment of new areas of brain because of plasticity after rehabilitation. 


\section{EARLY REHABILITATION}

In the NICU patients are alone in bed or bedside most of the time. There is an opportunity during intensive care for early mobilisation. Traditionally, bed rest has been prescribed after SAH due to the fear that head elevation in excess of $45^{\circ}$ could decrease cerebral perfusion and give rise to DCI. Mobilisation was feared to potentially cause re-bleeding from another unsecured aneurysm and negatively affect the development and course of cerebral vasospasm. The SAH patients can be mobilized early, it is safe and feasible. ${ }^{[40,41]}$ Physical mobilisation is the best evaluated example of early intervention. As soon as medically and neurologically advisable, the patient should be mobilised out of bed and encouraged to ambulate if feasible with help of a physiotherapist. The core component of the early rehabilitation intervention is the gradual increase in mobilisation level. The content of the early rehabilitation includes: mobilisation, prevention of contractures by passive exercises and adequate postural changes during rest and sleep, guidance in ADL and swallowing and eating, pulmonary rehabilitation, body exercises and balance training, reality orientation and information and emotional support to patients and their families. ${ }^{[41]}$ Overstimulation is avoided and breaks are provided between activities. Blood pressure is frequently monitored in both supine and vertical position. If there is orthostatic hypotension then mobilisation is delayed. Most of the patients, depending on their medical and neurological condition, can be mobilised next day after aneurysm clipping/coiling. The earlier and higher degree of mobilisation does not increase neurosurgical complications. Rather, the frequency and severity of cerebral vasospasm following SAH are alleviated and are not aggravated by early rehabilitation. Each step of mobilisation achieved during the first 4 days after surgery reduces the risk of severe vasospasm. ${ }^{[42]}$ If the patients are mobilised early then subtle neurological impairment due to cerebral vasospasm can be detected early, and prompt intervention can be done to prevent development of delayed ischemic neurological deficits. Early mobilization and rehabilitation probably increases the chance of a good functional outcome in poor grade SAH patients. ${ }^{[41]}$

Having observed the daily progress of each patient in the neurosurgery unit, the physiatrist can participate in discharge planning with the neurosurgeon. When the patient is medically stable and is considered psychologically and physically appropriate for intensive interdisciplinary rehabilitation, the physiatrist arranges for the patient's transfer to a rehabilitation ward. $^{[43]}$

\section{INPATIENT REHABILITATION}

The neurologic deficit that can be addressed in rehabilitation following SAH include: paralysis; aphasia, dysarthria, or both; dysphagia; cognitive perceptual impairment; sensory impairment; pain; visual perceptual impairment; spasticity; sphincter dysfunction; and social and vocational disability. The initial assessment can be done using systemically examining each of the following six systems: physical condition; upper extremities; lower extremities; sensory components related to speech, vision, and hearing system; sphincter function; and sensorium (mental and emotional status). Impairment in each system is scored from 1 indicating no gross abnormalities considering the age of the individual to 4 indicating severe abnormalities requiring constant assistance or supervision. The total score ranges from 6 to 24 . If the profile score is 6 , the patient has no functional impairment and probably does not require inpatient rehabilitation. If the profile score is 24 then the patient is too severely impaired to be admitted to a rehabilitation unit. ${ }^{[43]}$ Any patient with brain injury due to any disease who has persistent and stable neurological deficit, who requires medical monitoring and has impairment in at least two key domains should be transferred to in patient neurorehabilitation facility comprising of integrated neurorehabilitation team.

The integrated neurorehabilitation team should consist of:

- Rehabilitation physician (physiatrist)

- Neuropsychologist

- Speech and language pathologist

- Physical therapist

- Occupational therapist

- Rehabilitation nurse

- Orthotist

- Social worker

- Access to other medical specialties as best available in the setting.

\section{General principles}

- Initial functional evaluation should be done using FIM

- All team members should be trained in supported conversation to be able to interact with patients with communication limitations such as aphasia

- Patient, family and caregiver education is provided both formally and informally, with consideration given to individual and group settings as appropriate

- Patients should engage in training that is meaningful, engaging, progressively adapted, task specific and goal-oriented in an effort to enhance motor control and restore sensory motor function. Minimum $3 \mathrm{~h}$ of daily rehabilitation therapies should be given 
- Training should encourage the use of patient's involved affected limb during functional tasks and be designed to simulate partial or whole skills required in ADL (e.g., folding, buttoning, pouring and lifting)

- Therapists should provide supplementary training programs aimed at increasing the active movement and functional use of the affected arm between therapy sessions, e.g., Graded Repetitive Arm Supplementary Program (GRASP) suitable for use during hospitalisation and at home

- The GRASP should include: strengthening exercises for the arm and hand (using small wrist weight, putty, hand gripper), range of motion exercises (stretching, active exercises), and exercises that improve gross and fine motor skills (e.g., blocks)

- The need for special equipment (e.g., wheelchairs, safety devices) should be evaluated on an individual basis. Once provided, patients should be reassessed, as appropriate, to determine if changes are required or equipment can be discontinued with the aim of achieving normal unassisted function

- Spasticity and contractures can be prevented or treated by antispastic pattern positioning, range of motion exercises, and/or stretching

- Joint protection strategies should be used during the early or flaccid stage of recovery to prevent or minimise pain

- Assessment should be done weekly by team meeting for each patient.

\section{Management of each impairments}

\section{Paralysis}

Physical therapy

- Physical therapy starts with tilt table training administered to prevent orthostatic hypotension, stimulate antigravity muscle and improve body balance

- Range of movement exercise for the affected upper and lower extremities are administered

- Strengthening exercises for the unaffected side are also emphasised because the patient needs to depend on that side in ADL more than before the SAH

- When body balance and motor function of the affected lower extremity improve at least proximally, perambulation training is started in parallel bar

- As the patient improves in balance and in forward motion of the affected lower extremity ambulation is advanced

- A walker is often used if the affected upper limb shows functional strength of the hand

- If the ankle dorsiflexion power is <3/5 then the patient is fitted with a custom-moulded ankle-foot orthosis to prevent foot drop during ambulation
- The physical therapists also train the patient in transfer skills and bathroom activities.

\section{Occupation therapy}

- In occupational therapy, therapeutic exercises for the affected upper extremity are provided. In addition the patient is taught to use any remaining upper limb function for personal hygiene, grooming, feeding and dressing activities

- As progress is made in self-care activities, homemaking and prevocational training are initiated

- A hand splint when needed to prevent flexion contracture of the wrist and fingers may also fabricated.

\section{Spasticity}

Spasticity can be troublesome and may influence the outcome of rehabilitation negatively after SAH. Spasticity is a common symptom, which requires intervention, especially when it interferes with functional abilities such as mobility, positioning or hygiene, or when it is the cause of deformity or pain. The common drugs used for treatment of spasticity are baclofen and tizanidine. Baclofen appears to improve lower extremity spasticity, and its starting dose is $5 \mathrm{mg}$ tid to maximum dose $80 \mathrm{mg} /$ day. Baclofen may lower seizure threshold. Tizanidine is effective for improving upper and lower extremity spasticity, and its starting dose is $4 \mathrm{mg}$ to maximum dose $36 \mathrm{mg} /$ day. Tizanidine may be hepatotoxic. Referral to a specialist is required if the spasticity cannot be managed with oral medications for following indications.

Local spasticity

- Botulinum toxin type A injections

- Phenol blocks of the musculoskeletal nerve

- Electrical stimulation.

Generalised spasticity not responding to oral baclofen or if patient cannot tolerate oral baclofen.

- Intrathecal baclofen:

- Reduces upper and lower extremity spasticity

- Improves walking performance.

\section{Speech and language}

When there are communication difficulties resulting from aphasia or dysphasia, a cognitive linguistic impairment or both, a speech pathologist should evaluate the patient to determine the severity of the deficits in verbal expression, auditory comprehension reading, writing, and gesturing. A clear diagnosis of the speech disorder type can then be made.

When speech deficit are identified, supportive speech therapy is administered to the patient. Although the efficacy of speech therapy remain controversial it is applied whenever available to provide the programmed 
stimulation necessary to use and maximise any residual speech ability. ${ }^{[43]}$

Because of physiologic and spontaneous restitution, most patients with aphasia undergoing rehabilitation have some measurable spontaneous speech recovery with time.

Strategies to help patient understand:

- Make sure you have the person's attention before speaking

- Say name and make sure patient is looking at you

- Speak slowly

- Try to use the simplest words that can communicate your message

- Use short sentences. Repeat your sentence if necessary

- Use pictures, gestures and demonstrations to help him/her to understand your words.

Strategies to help the person with aphasia to speak the correct words:

- Help to practice words by naming objects around the house

- Encourage to use gestures

- Ask to describe what an object looks like or what it is used for

- Even if patient leaves out words in a sentence, patient may be able to communicate his/her message. Repeat the full sentence back to so person can hear the words that he/she did not include in the sentence

- If person is unable to say a word that patient wants to say, ask to watch and listen to you as you say the word. Then ask to repeat the word

- Ask to practice words and phrases that are most often needed. For example, 'I have pain', 'I want water', etc.

Problems in speaking clearly (dysarthria):

- Slurred speech sounds

- Speech too fast, too slow or too soft

- The voice may have a weak, strained or hoarse quality

- If the patients talks too fast, ask them to say one word at a time. You may ask the patients to tap fingers for each word that they want to say. Tapping helps to create a slow rhythm

- If a patient talks too slowly, give time to talk. This may be the only way person can make herself understood

- If voice is very soft or weak, ask to take a deep breath before starting to talk

- Following exercises may help person to speak clearly: Smile exercise, kissing exercise, and tongue exercise

- Therapy for dysarthria is aimed at guiding the patient to produce sounds, then word and then entire sentence. In severe cases of unintelligible dysarthria, other communication methods may be explored such as writing, typing, use of a communication instrument pictures, or sign language.

Reading:

- Check if the patient is able to read, comprehend and answer written commands/directions/signs accurately

- Ascertain that patient does not complains of headache or double vision when reading

- Ask the patient to read single words or short phrases and gradually increase the number of words to be read

- Instruct the patient to take the finger and move it from one word to the next

- If the patient has double vision, cover one eye with a patch

- If the patient has difficulty concentrating, provide a quiet place for reading.

Writing:

- Observe if the patient can write his name, address, simple numbers, simple words or notes and make them practice

- Relearning is a slow process and would require patience, and one step at a time approach.

\section{Dysphagia}

A brain injury can cause both physical and thinking difficulties that interfere with the ability to swallow. Dysphagia is common in patients with stroke particularly patients with aphasia, but its incidence after SAH alone is not well documented in the literature. During the first 3 months after an SAH swallowing function may return spontaneously as the neurologic condition improves. Meanwhile compensatory swallowing technique, for eample, chin tuck, head turn, and multiple swallow to reduce aspiration can be applied during oral feeding at the bedside. However, the use of the compensatory technique may not reduce dysphagia-related medical complications. ${ }^{[4]]}$ If dysphagia is persisting it should be evaluated by a swallowing disorder therapist, who is a especially trained speech pathologist or occupational therapist. If severe dysphagia is expected to last a long time, a percutaneous endoscopic gastrostomy feeding tube is inserted for proper hydration and daily nutritional support. In the rehabilitation hospital setting, the video-modified barium swallow evaluation may be repeated every 2 weeks to reassess progress in swallowing function. ${ }^{[43]}$

Tips for managing patients with difficulty in swallowing:

- Make sure the patient is sitting upright. Support head if necessary

- Encourage to take one sip/bite at a time

- Help the patient to lower chin down toward chest during swallowing 
- Try giving liquids by spoon or straw

- Ask the patient to swallow two times for each sip/bite

- Ask the patient to take a breath and hold it, then swallow and breathe

- Check between swallows and after the meal to make sure there is no food left in the mouth

- Offer one item of food at a time

- Do not talk while eating or drinking

- Start with baby food, e.g., halwa, khichdi, banana, ganjee, etc.

- A helping person should remain with patient to assure that patient eats and drinks safely

- Assist patient to sit upright for 30-60 min after eating

- Patients with cognitive difficulty in feeding may require instruction as given to small children with modification of utensils.

\section{Sphincter dysfunction}

Urinary incontinence is frequently encountered during acute rehabilitation of SAH survivor particularly in patient who demonstrate significant cognitive dysfunction. The commonest cause is uninhibited neurogenic bladder. To manage these patients, anticholinergic medication unless medically contraindicated, daily monitoring of urinary post-void residual, and a trial of self-voiding can be used. If urinary incontinence results only from confusion and toileting is not possible then condom external catheters can be applied for male, and clean intermittent catheterisation can be performed for females by rehabilitation nurse every $6 \mathrm{~h}$. When urinary retention occur, obstruction of the bladder outlet in male and medication-related anticholinergic side effect should be ruled out. While a causative factor is being investigated, intermittent urinary catheterisation is started. It is important to identify and manage early urinary tract infection using short-term oral antibiotic. ${ }^{[43]}$

\section{COGNITIVE REHABILITATION THERAPY}

Cognitive rehabilitation therapy (CRT) can be carried out at all stages of recovery (acute, subacute, and post-acute), and in various settings (in-patient, out-patient and domestic environments). It can be administered in different modalities (individual, family, and group). The CRT involves professional in different disciplines (neuropsychologists, occupational therapists, and speech and language pathologists). CRT can be effective regardless of the length of time since injury, and the injury severity.

Graduates with job requiring high level of intelligence may have severe disability due to cognitive impairment.

- Such patients should be referred for comprehensive neuropsychological assessment and cognitive retraining therapy at special centres
- Hierarchy of CRT should address attention followed by memory then executive functions

- A mix of restoration and compensation approaches should be used

- The therapy should be systematic, structured and repetitive according to the needs of each particular patient.

Patients with poor education status and not so demanding job can be managed at home with following tasks:

- Improving mental speed:

- Sorting of grains/beads of different colors and sizes.

- Improving attention:

- Letter cancellation/number cancellation/ cancellation of shapes

- Copywriting and dictation.

- Executive functions and working memory:

- Mental calculation

- Reciting poetry

- Reciting arithmetic table

- Remembering shopping list

- Remembering new routes

- Categorisation of household articles

- Planning for social events.

- Visuospatial functions:

- Copying drawings

- Learning rangoli

- Comparing pictures.

\section{ENDOCRINE DYSFUNCTION}

Neuroendocrine dysfunction is not uncommon after $\mathrm{SAH}$. The prevalence of pituitary dysfunction is more in the acute phase and decreases in chronic phase. Abnormalities in basal hormonal levels are more frequent when compared to induction tests, and the prevalence of a single pituitary hormone dysregulation is more frequent than multiple hormone dysregulation. These abnormalities have not been shown to consistently impact outcome. There is a need for well-designed prospective studies to more precisely assess the incidence, clinical course, and outcome impact of pituitary dysfunction after $\mathrm{SAH} .{ }^{[45]}$

\section{EFFICACY OF REHABILITATION FOR SUBARACHNOID HAEMORRHAGE}

$\mathrm{SAH}$ patients receiving in-patient rehabilitation make functional gains, although the rate of gain is less than for TBI or stroke. SAH patients tend to have longer rehabilitation ward stays compared to stroke or TBI patients but no significant difference in living arrangements or FIM. Longer length of stay (LOS) could be explained by several factors including slower rate of recovery for $\mathrm{SAH}$, more medical complications and lower 
functional level on admission. Furthermore, the FIM may not reflect cognitive processing difficulties, which have a significant impact on independence and rehabilitation LOS. ${ }^{[27]}$ The presence of hydrocephalus negatively impacts on outcome. The majority of SAH survivors who receive inpatient rehabilitation attain physical independence, but many continue to have cognitive impairments which result in social and vocational disabilities. ${ }^{[3]}$ The severity of cognitive impairment has predictive value for the functional status and the level of supervision required at discharge. ${ }^{[46]}$ It has also been found that social, behavioural and cognitive deficits are relatively common after SAH and have significant impact of work re-entry, home and community integration. Personality changes have been found to be a great hindrance to functional social reintegration. ${ }^{[27]}$ Early mobilisation through aggressive rehabilitation programs (originally thought to improve only motor and physical outcomes) has been shown to improve cognitive function as well. ${ }^{[47,48]}$ As a general rule everything improves during the first 18 months but perhaps there is benefit in following up patients up to 5 years after SAH. ${ }^{[49]} \mathrm{SAH}$ patients do well in the acute rehabilitation setting and this is the most appropriate setting for these individuals, as they tend to make substantial functional recovery. The process of distinguishing which rehabilitation treatments will provide the best outcomes for patients with a specific constellation of deficits is still evolving.

\section{Financial support and sponsorship Nil.}

\section{Conflicts of interest}

There are no conflicts of interest.

\section{REFERENCES}

1. Al-Shahi R, White PM, Davenport RJ, Lindsay KW. Subarachnoid haemorrhage. BMJ 2006;333:235-40.

2. Hop JW, Rinkel GJ, Algra A, van Gijn J. Case-fatality rates and functional outcome after subarachnoid hemorrhage: A systematic review. Stroke 1997;28:660-4.

3. Lovelock CE, Rinkel GJ, Rothwell PM. Time trends in outcome of subarachnoid hemorrhage: Population-based study and systematic review. Neurology 2010;74:1494-501.

4. van Gijn J, Rinkel GJ. Subarachnoid haemorrhage: Diagnosis, causes and management. Brain 2001;124(Pt 2):249-78.

5. Jaja BN, Lingsma H, Steyerberg EW, Schweizer TA, Thorpe KE, Macdonald RL, et al. Neuroimaging characteristics of ruptured aneurysm as predictors of outcome after aneurysmal subarachnoid hemorrhage: Pooled analyses of the SAHIT cohort. J Neurosurg 2016;124:1703-11.

6. Diringer MN, Edwards DF. Admission to a neurologic/ neurosurgical Intensive Care Unit is associated with reduced mortality rate after intracerebral hemorrhage. Crit Care Med 2001;29:635-40.

7. Josephson SA, Douglas VC, Lawton MT, English JD, Smith WS, Ko NU. Improvement in Intensive Care Unit outcomes in patients with subarachnoid hemorrhage after initiation of neurointensivist co-management. J Neurosurg 2010;112:626-30.
8. Molyneux AJ, Kerr RS, Yu LM, Clarke M, Sneade M, Yarnold JA, et al. International Subarachnoid Aneurysm Trial (ISAT) of neurosurgical clipping versus endovascular coiling in 2143 patients with ruptured intracranial aneurysms: A randomised comparison of effects on survival, dependency, seizures, rebleeding, subgroups, and aneurysm occlusion. Lancet 2005;366:809-17.

9. Jaja BN, Cusimano MD, Etminan N, Hanggi D, Hasan D, Ilodigwe D, et al. Clinical prediction models for aneurysmal subarachnoid hemorrhage: A systematic review. Neurocrit Care 2013;18:143-53.

10. Risselada R, Lingsma HF, Bauer-Mehren A, Friedrich CM, Molyneux AJ, Kerr RS, et al. Prediction of 60 day case-fatality after aneurysmal subarachnoid haemorrhage: Results from the International Subarachnoid Aneurysm Trial (ISAT). Eur J Epidemiol 2010;25:261-6.

11. le Roux AA, Wallace MC. Outcome and cost of aneurysmal subarachnoid hemorrhage. Neurosurg Clin $N$ Am 2010;21:235-46.

12. Lanzino G, Kassell NF, Germanson TP, Kongable GL, Truskowski LL, Torner JC, et al. Age and outcome after aneurysmal subarachnoid hemorrhage: Why do older patients fare worse? J Neurosurg 1996;85:410-8.

13. Jaja BN, Lingsma H, Schweizer TA, Thorpe KE, Steyerberg EW, Macdonald RL; SAHIT Collaboration. Prognostic value of premorbid hypertension and neurological status in aneurysmal subarachnoid hemorrhage: Pooled analyses of individual patient data in the SAHIT repository. J Neurosurg 2015;122:644-52.

14. Bulters DO, Santarius T, Chia HL, Parker RA, Trivedi R, Kirkpatrick PJ, et al. Causes of neurological deficits following clipping of 200 consecutive ruptured aneurysms in patients with good-grade aneurysmal subarachnoid haemorrhage. Acta Neurochir (Wien) 2011;153:295-303.

15. Cedzich C, Roth A. Neurological and psychosocial outcome after subarachnoid haemorrhage, and the hunt and hess scale as a predictor of clinical outcome. Zentralbl Neurochir 2005;66:112-8.

16. Ogden JA, Mee EW, Henning $M$, Smith RR, Haines SJ. A prospective study of impairment of cognition and memory and recovery after subarachnoid hemorrhage. Neurosurgery 1993;33:572-87.

17. Mahaney KB, Todd MM, Bayman EO, Torner JC; IHAST Investigators. Acute postoperative neurological deterioration associated with surgery for ruptured intracranial aneurysm: Incidence, predictors, and outcomes. J Neurosurg 2012;116:1267-78.

18. Wong GK, Lam SW, Ngai K, Wong A, Siu D, Poon WS, et al. Cognitive domain deficits in patients with aneurysmal subarachnoid haemorrhage at 1 year. J Neurol Neurosurg Psychiatry 2013;84:1054-8.

19. Stienen MN, Weisshaupt R, Fandino J, Fung C, Keller E, Hildebrandt G, et al. Current practice in neuropsychological outcome reporting after aneurysmal subarachnoid haemorrhage. Acta Neurochir (Wien) 2013;155:2045-51.

20. Al-Khindi T, Macdonald RL, Schweizer TA. Cognitive and functional outcome after aneurysmal subarachnoid hemorrhage. Stroke 2010;41:e519-36.

21. Haug T, Sorteberg A, Sorteberg W, Lindegaard KF, Lundar T, Finset A. Cognitive outcome after aneurysmal subarachnoid hemorrhage: Time course of recovery and relationship to clinical, radiological, and management parameters. Neurosurgery 2007;60:649-56.

22. Suarez JI. Timing of neuropsychological outcome measures in patients with subarachnoid hemorrhage. Stroke 2007;38:1724-5.

23. Dey S, Kumar KJ, Shukla D, Bhat D. Neurological, 
neuropsychological, and functional outcome after good grade aneurysmal subarachnoid haemorrhage. Neurol India 2017. [In Press].

24. Hütter BO, Kreitschmann-Andermahr I, Gilsbach JM. Cognitive deficits in the acute stage after subarachnoid hemorrhage. Neurosurgery 1998;43:1054-65.

25. Shukla D, Devi BI, Agrawal A. Outcome measures for traumatic brain injury. Clin Neurol Neurosurg 2011;113:435-41.

26. Rao S, Subbukrishna D, Kumar G. NIMHANS Neuropsychology Battery Manual. Bangalore: NIMHANS; 2004.

27. Stern M, Chang D, Odell M, Sperber K. Rehabilitation implications of non-traumatic subarachnoid haemorrhage. Brain Inj 2006;20:679-85.

28. Caeiro L, Santos CO, Ferro JM, Figueira ML. Neuropsychiatric disturbances in acute subarachnoid haemorrhage. Eur J Neurol 2011;18:857-64.

29. Sanchez CE, Ogilvy CS, Carter BS. Outcomes studies in cerebrovascular neurosurgery. Neurosurg Focus 2007;22:E11.

30. NIHSS. NIH Stroke Scale. Available from: http://www. ninds.nih.gov/doctors/nih_stroke_scale.pdf. [Last cited on 2012 Dec 21].

31. Huybrechts KF, Caro JJ. The Barthel Index and modified Rankin Scale as prognostic tools for long-term outcomes after stroke: A qualitative review of the literature. Curr Med Res Opin 2007;23:1627-36.

32. Sulter G, Steen C, De Keyser J. Use of the Barthel index and modified Rankin scale in acute stroke trials. Stroke 1999;30:1538-41.

33. Heinemann AW, Linacre JM, Wright BD, Hamilton BB, Granger C. Relationships between impairment and physical disability as measured by the functional independence measure. Arch Phys Med Rehabil 1993;74:566-73.

34. Dombovy ML, Drew-Cates J, Serdans R. Recovery and rehabilitation following subarachnoid haemorrhage: Part II. Long-term follow-up. Brain Inj 1998;12:887-94.

35. Report of World Federation of Neurological Surgeons Committee on a universal subarachnoid hemorrhage grading scale. J Neurosurg 1988;68:985-6.

36. Pettigrew LE, Wilson JT, Teasdale GM. Assessing disability after head injury: Improved use of the Glasgow Outcome Scale. J Neurosurg 1998;89:939-43.

37. Passier PE, Visser-Meily JM, Rinkel GJ, Lindeman E, Post MW Determinants of health-related quality of life after aneurysmal subarachnoid hemorrhage: A systematic review. Qual Life Res 2013;22:1027-43.
38. Traumatic Brain Injury: Multi Organizational Consensus Recommendations for India; 2015. Available from: http:// www.ntsi.co.in/Version.pdf. [Last cited on 2016 May 03].

39. Kelly C, Foxe JJ, Garavan H. Patterns of normal human brain plasticity after practice and their implications for neurorehabilitation. Arch Phys Med Rehabil 2006;87 12 Suppl 2:S20-9.

40. Olkowski BF, Devine MA, Slotnick LE, Veznedaroglu E, Liebman KM, Arcaro ML, et al. Safety and feasibility of an early mobilization program for patients with aneurysmal subarachnoid hemorrhage. Phys Ther 2013;93:208-15.

41. Karic T, Røe C, Nordenmark TH, Becker F, Sorteberg A. Impact of early mobilization and rehabilitation on global functional outcome one year after aneurysmal subarachnoid hemorrhage. J Rehabil Med 2016;48:676-82.

42. Karic T, Røe C, Nordenmark TH, Becker F, Sorteberg A. Effect of early mobilization and rehabilitation on complications in aneurysmal subarachnoid hemorrhage. J Neurosurg 2016:1-9. [Epub ahead of print], PubMed PMID: 27058204.

43. Ahn JH. Rehabilitation after subarachnoid hemorrhage. In: Le Roux PD, Winn HR, Newell DW, editors. Management of Cerebral Aneurysms. Philadelphia: Saunders; 2004. p. $499-506$.

44. DePippo KL, Holas MA, Reding MJ, Mandel FS, Lesser ML. Dysphagia therapy following stroke: A controlled trial. Neurology 1994;44:1655-60.

45. Robba C, Bacigaluppi S, Bragazzi N, Lavinio A, Gurnell M, Bilotta $\mathrm{F}$, et al. Clinical prevalence and outcome impact of pituitary dysfunction after aneurysmal subarachnoid hemorrhage: A systematic review with meta-analysis. Pituitary 2016;19:522-35.

46. Saciri BM, Kos N. Aneurysmal subarachnoid haemorrhage: Outcomes of early rehabilitation after surgical repair of ruptured intracranial aneurysms. J Neurol Neurosurg Psychiatry 2002;72:334-7.

47. Shimamura N, Matsuda N, Satou J, Nakano T, Ohkuma H. Early ambulation produces favorable outcome and nondemential state in aneurysmal subarachnoid hemorrhage patients older than 70 years of age. World Neurosurg 2014;81:330-4.

48. Kara B, Yozbatiran N, Arda MN. Functional results of physiotherapy programme on patients with aneurysmal subarachnoid hemorrhage. Turk Neurosurg 2007;17:83-90.

49. Cheng H, Shi J, Zhou M. Cognitive assessment in Chinese patients with aneurysmal subarachnoid hemorrhage. Behav Neurol 2006;17:117-20. 